\title{
SOLVING LINEAR PROGRAMMINGPROBLEM BYINVERSE OF SUB MATRIX
}

\author{
Jaafer Hmod Eidi \& Saad Shakir Mahmood \\ saadshakirmahmood@yahoo.com \\ Department of Mathematics, College of Education, University of Mustansiriyah
}

\begin{abstract}
The aim of this paper is to propose a new technique to solve the Linear Programming Problem by converting the constraints to the formula of the 1 and all the coefficients of the objective function to 1 say the one formulalinear programming problem and then the system solved by inverse of sub matrix coefficients.

Keywords: Linear Programming, simplex method, sub matrix.
\end{abstract}

\section{Introduction}

Simplex algorithm represents the most effect method to solve the linear programming problem by converting the problem to the canonical or standard form and then solve it by using the elimination technique to change the basic variables with a new basic variables. In simplex method the number of variables in the given system must be less than the total number of variables in canonical form where the slack variables must be added to the system for inequality less than or equal and the surpluses variables must be subtract from the inequality greater than or equal, and hence must be added an artificial variables to guarantee the existents of the basic variables. Our proposed method makes deal with matrix only and there are no any added variables and no elimination proses and without comparison between the ratios which make the proses of solution is very simple.

\section{Solving Linear Programming Problem (LPP) by inverse of sub matrix}

Given the following LPP

S.T. $\quad A x \leq b$

$$
\max . z=c^{T} x
$$

$$
x_{i} \geq 0, \forall i=1,2, \ldots, n \text {, where } x, c \in R^{n}, b \in R^{m}, A \in R^{n x m}
$$

Before the analysis of our proposed method the following definitions are needed:

\section{Definition 1:}

The constraints is called in the 1 formulaif all Right- hand sides of this constraints are equal to 1 .

\section{Definition 2:}

For a matrix A, column $i$ is called dominated by column $j$ if $a_{k i} \geq a_{k j}, \forall k$, in this case the column $i$ can be deleted

\section{Definition 3:}

For a matrix A, row $i$ is called dominated by row $j$ if $a_{i k} \geq a_{j k}, \forall k$, in this case the row $i$ can be deleted

LPP (1) can be wright as follows: 
$\max . z=c_{1} x_{1}+c_{2} x_{2}+\cdots+c_{n} x_{n}$

S.T. $\quad a_{11} x_{1}+a_{12} x_{2}+\cdots+a_{1 n} x_{n} \leq b_{1}$

$$
\begin{gathered}
a_{21} x_{1}+a_{22} x_{2}+\cdots+a_{2 n} x_{n} \leq b_{2} \\
\cdot \cdot \cdot \cdot \cdot \cdot \\
\cdot \cdot \cdot \cdot \cdot \cdot \cdot \\
a_{m 1} x_{1}+a_{m 2} x_{m 2}+\cdots a_{m n} x_{n} \leq b_{m} \\
\quad x_{i} \geq 0, \quad \forall i
\end{gathered}
$$

The first step is to convert the LPP to the one form as follows:

Suppose $y_{i}=c_{i} x_{i}, \forall i$

The optimal solution of the LPP is consisting of $m(m \leq n)$ basic variables or consisting of $n(n \leq m)$ basic variables and hence the another variables must be zero.In this case the sub matrix must be of order $m x m(m \leq n)$ or of order $n x n(n \leq m)$, and hence the optimal solution must be the exact solution of only one sub matrix where the exact solution is the inverse of this sub matrix.

All the RHS of the constraints must become one,by divided the j-thconstraintsby $b_{j}, \forall j=$ $1,2, \ldots, m$

The LPP (2) becomes as follows:

$\max . z=y_{1}+y_{2}+\cdots+y_{n}$

S.T. $\quad d_{11} y_{1}+d_{12} y_{2}+\cdots+d_{1 n} y_{n} \leq 1$

$$
d_{21} y_{1}+d_{22} y_{2}+\cdots+d_{2 n} y_{n} \leq 1
$$

$d_{m 1} y_{1}+d_{m 2} y_{2}+\cdots+d_{m n} y_{n} \leq 1$

$y_{i} \geq 0, \forall i$ and $d_{j i}=\frac{a_{j i}}{b_{j} c_{i}}, \forall j, i$

The second step is to deal with the matrix coefficients as follows:

$A=\left[\begin{array}{cccccc}d_{11} & d_{12} & \cdot & \cdot & \cdot & d_{1 n} \\ d_{21} & d_{22} & \cdot & \cdot & \cdot & d_{2 n} \\ \cdot & \cdot & \cdot & \cdot & \cdot & \cdot \\ : & : & : & : & : & : \\ d_{m 1} & d_{m 2} & \cdot & \cdot & \cdot & d_{m n}\end{array}\right]$

Suppose that $A_{k m}$ is all the possible sub-matrices of the matrix $\mathrm{A}$, where the meaning of sub matrix is any $m$ row from $A$ and any m column from $A$ which can representsmxm matrix and $\mathrm{k}$ be the sub matrix number.

Then, the solution of (4) is given in the following formula:

$y_{i}=\sum_{j=1}^{m} S_{j i}, i=1,2, \ldots, n$, where $S=\left(A_{m}\right)^{-1} \quad$ which satisfy all constraints and have optimal value of the objective function.

The following examples explain the proses of solution:

\section{Example 1:[1]}

S.T. $\quad 2 x_{1}+3 x_{2}+5 x_{4} \leq 12$

$$
\max . z=3 x_{1}+x_{2}+2 x_{3}+4 x_{4}
$$

$4 x_{1}+2 x_{3}-3 x_{4} \leq 10$

Suppose $y_{1}=3 x_{1}, y_{2}=x_{2}, y_{3}=2 x_{3}$, and $y_{4}=4 x_{4}$ 
The given LPP becomes as follows:

$\max . z=y_{1}+y_{2}+y_{3}+y_{4}$

S.T. $\frac{1}{18} y_{1}+\frac{1}{4} y_{2}+\frac{5}{48} y_{4} \leq 1$

$$
\begin{gathered}
\frac{2}{15} y_{1}+\frac{1}{10} y_{3}-\frac{3}{40} y_{4} \leq 1 \\
y_{1}, y_{2}, y_{3}, \text { and } y_{4} \geq 0
\end{gathered}
$$

The coefficients matrix $A=\left[\begin{array}{cccc}\frac{1}{18} & \frac{1}{4} & 0 & \frac{5}{48} \\ \frac{2}{15} & 0 & \frac{1}{10} & \frac{-3}{40}\end{array}\right]$

All sub matrix are $A_{12}=\left[\begin{array}{cc}\frac{1}{18} & \frac{1}{4} \\ \frac{2}{15} & 0\end{array}\right]$, and $A_{12}^{-1}=\left[\begin{array}{cc}0 & 7.5 \\ 4 & -1.6667\end{array}\right]$,

Given $y_{1}=0+7.5=7.5$ and $y_{2}=4-1.6667=2.3333, z=9.8333 A_{22}=\left[\begin{array}{cc}\frac{1}{18} & 0 \\ \frac{2}{15} & \frac{1}{10}\end{array}\right]$, given $y_{1}=18$ and $y_{3}=-14$ is not feasible $A_{32}=\left[\begin{array}{cc}\frac{1}{8} & \frac{5}{48} \\ \frac{2}{15} & \frac{-3}{40}\end{array}\right]$,

given $y_{1}=9.9231$ and $y_{4}=4.3077, \quad z=14.2308$

$A_{42}=\left[\begin{array}{cc}\frac{1}{4} & 0 \\ 0 & \frac{1}{10}\end{array}\right]$, given $y_{2}=4$ and $y_{3}=10, z=14 A_{52}=\left[\begin{array}{cc}\frac{1}{4} & \frac{5}{48} \\ 0 & \frac{-3}{40}\end{array}\right]$,

given $y_{2}=4$ and $y_{4}=-7.7778$ is not feasible $A_{62}=\left[\begin{array}{cc}0 & \frac{5}{48} \\ \frac{1}{10} & \frac{-3}{40}\end{array}\right]$,

given $y_{3}=17.2$ and $y_{4}=9.6, z=26.8$

And hence the optimal solution is $y_{3}=17.2$, and $y_{4}=9.6, z=26.8$

Which mean that the optimal solution for the original LP is

$$
x_{1}=0, x_{2}=0, x_{3}=8.6, x_{4}=2.4, \text { and } z=26.8
$$

\section{Example 2:[2]}

$\max . z=x_{1}+2 x_{2}+3 x_{3}+4 x_{4}$

S.T. $\quad 4 x_{1}-2 x_{2}-4 x_{3}-x_{4} \leq 1$

$$
\begin{aligned}
& 3 x_{1}+x_{2}+x_{3}+2 x_{4} \leq 1 \\
& 2 x_{1}+3 x_{2}-2 x_{3}-2 x_{4} \leq 1 \\
& -x_{1}-3 x_{2}-3 x_{3}+x_{4} \leq 1 \\
& x_{1}, x_{2}, x_{3}, x_{4} \geq 0
\end{aligned}
$$

Since the RHS of the constrained are all same equal to one and one can see that columns 1,2 , and 4 dominated by column 3 , and hence the columns 1,2, and 4 can be deleted and the LP becomes

$\max . z=3 x_{3}$

S.T. $\quad-4 x_{3} \leq 1$

$$
\begin{gathered}
x_{3} \leq 1 \\
-x_{3} \leq 1 \\
-3 x_{3} \leq 1 \\
x_{3} \geq 0
\end{gathered}
$$

Hence $A_{11}=[-4]$, given $x_{3}=-\frac{1}{4}$ is not feasible 
$A_{21}=[1]$, given $x_{3}=1, \mathrm{z}=3$

$A_{31}=[-2]$, given $x_{3}=-\frac{1}{2}$ is not feasible

$A_{41}=[-3]$, given $x_{3}=-\frac{1}{3}$ is not feasible

And the optimal solution is $x_{1}=0, x_{2}=0, x_{3}=1, x_{4}=0$, and $z=3$.

Consider the following LPP:

$\min . z=c_{1} x_{1}+c_{2} x_{2}+\cdots+c_{n} x_{n}$

S.T. $\quad a_{11} x_{1}+a_{12} x_{2}+\cdots+a_{1 n} x_{n} \geq b_{1}$

$$
a_{21} x_{1}+a_{22} x_{2}+\cdots+a_{2 n} x_{n} \geq b_{2}
$$

. . . . . . . . . .

$$
\begin{gathered}
a_{m 1} x_{1}+a_{m 2} x_{m 2}+\cdots a_{m n} x_{n} \geq b_{m} \\
x_{i} \geq 0, \quad \forall i
\end{gathered}
$$

The first step is to convert the LP to the one form as follows:

Suppose $y_{i}=c_{i} x_{i}, \forall i$

All RHS of the constrained must become one by divided the $\mathrm{j}$-th constrained by $b_{j}, \forall j=$ $1,2, \ldots, m$

The LP (6) become as follows:

$\min . z=y_{1}+y_{2}+\cdots+y_{n}$

S.T. $\quad d_{11} y_{1}+d_{12} y_{2}+\cdots+d_{1 n} y_{n} \geq 1$

$$
d_{21} y_{1}+d_{22} y_{2}+\cdots+d_{2 n} y_{n} \geq 1
$$

$d_{m 1} y_{1}+d_{m 2} y_{2}+\cdots+d_{m n} \dot{y}_{n} \geq 1$

$y_{i} \geq 0, \forall i$ and $d_{j i}=\frac{a_{j i}}{b_{j}}, \forall j, i$

The second step is to deal with the matrix coefficients as follows:

$A=\left[\begin{array}{cccccc}d_{11} & d_{12} & \cdot & \cdot & \cdot & d_{1 n} \\ d_{21} & d_{22} & \cdot & \cdot & \cdot & d_{2 n} \\ \cdot & \cdot & \cdot & \cdot & \cdot & \cdot \\ : & : & : & : & : & : \\ d_{m 1} & d_{m 2} & \cdot & \cdot & \cdot & d_{m n}\end{array}\right]$

Suppose that $A_{k m}$ is all the possible sub matrix of the matrix A, where the meaning of sub matrix is any $m$ row from $A$ and any m column from $A$ which can represent mxm matrix and $\mathrm{k}$ be the sub matrix number.

And the solution of (4) is given in the following formula:

$y_{i}=\sum_{j=1}^{m} S_{j i}, i=1,2, \ldots, n$, where $S=\left[\left(A_{m}\right)^{-1}\right]^{T}$ which satisfy all constrained and have optimal value of the objective function.

The following examples explain the proses of solution:

\section{Example 3:[3]}

S.T. $\quad 2 x_{1}+3 x_{2}+5 x_{4} \geq 12$

$$
\text { min. } z=3 x_{1}+x_{2}+2 x_{3}+4 x_{4}
$$

$$
4 x_{1}+2 x_{3}-3 x_{4} \geq 10
$$

$$
x_{1}, x_{2}, x_{3}, x_{4} \geq 0
$$

Suppose $y_{1}=3 x_{1}, y_{2}=x_{2}, y_{3}=2 x_{3}$, and $y_{4}=4 x_{4}$ 
The given LP becomes as follows:

$\min . z=y_{1}+y_{2}+y_{3}+y_{4}$

S.T. $\frac{1}{18} y_{1}+\frac{1}{4} y_{2}+\frac{5}{48} y_{4} \geq 1$

$$
\begin{gathered}
\frac{2}{15} y_{1}+\frac{1}{10} y_{3}-\frac{3}{40} y_{4} \geq 1 \\
y_{1}, y_{2}, y_{3}, \text { and } y_{4} \geq 0
\end{gathered}
$$

The coefficients matrix $A=\left[\begin{array}{cccc}\frac{1}{18} & \frac{1}{4} & 0 & \frac{5}{48} \\ \frac{2}{15} & 0 & \frac{1}{10} & \frac{-3}{40}\end{array}\right]$

All sub matrix are $A_{12}=\left[\begin{array}{cc}\frac{1}{8} & \frac{1}{4} \\ \frac{2}{15} & 0\end{array}\right], A_{12}^{-1}=\left[\begin{array}{cc}0 & 7.5 \\ 4 & -1.6667\end{array}\right]$,

given $y_{1}=0+4=4$ and $y_{2}=7.5-1.6667=5.8333, z=9.8333 A_{22}=\left[\begin{array}{cc}\frac{1}{18} & 0 \\ \frac{2}{15} & \frac{1}{10}\end{array}\right]$,

given $y_{1}=-6$ and $y_{3}=10$ is not feasible $A_{32}=\left[\begin{array}{cc}\frac{1}{8} & \frac{5}{48} \\ \frac{2}{15} & \frac{-3}{40}\end{array}\right]$,

given $y_{1}=11.5384$ and $y_{4}=2.6923, z=14.2308 A_{42}=\left[\begin{array}{cc}\frac{1}{4} & 0 \\ 0 & \frac{1}{10}\end{array}\right]$,

given $y_{2}=4$ and $y_{3}=10, z=14 A_{52}=\left[\begin{array}{cc}\frac{1}{4} & \frac{5}{48} \\ 0 & \frac{-3}{40}\end{array}\right]$,

given $y_{2}=9.5556$ and $y_{4}=-13.333$ is not feasible $A_{62}=\left[\begin{array}{cc}0 & \frac{5}{48} \\ \frac{1}{10} & \frac{-3}{40}\end{array}\right]$,

given $y_{3}=17.2$ and $y_{4}=9.6, z=26.8$

And hence the optimal solution is $y_{1}=4$, and $y_{2}=5.8333, z=9.8333$

Which mean that the optimal solution for the original LP is

$$
x_{1}=2.5, x_{2}=2.3333, x_{3}=0, x_{4}=0 \text {, and } z=9.8333
$$

\section{Example 4:[4]}

min. $z=x_{1}+2 x_{2}+3 x_{3}+4 x_{4}$

S.T. $\quad 4 x_{1}+3 x_{2}+2 x_{3}-x_{4} \geq 1$

$-2 x_{1}+x_{2}+3 x_{3}-3 x_{4} \geq 1$

$-4 x_{1}+x_{2}-2 x_{3}-3 x_{4} \geq 1$

$-x_{1}+2 x_{2}-2 x_{3}+x_{4} \geq 1$

$x_{1}, x_{2}, x_{3}, x_{4} \geq 0$

Since the RHS of the constrained are all same equal to one and one can see that rows 1,2 , and 4 dominated by row 3 , and hence the constraints 1,2 , and 4 can be deleted and the LPP becomes

min. $z=x_{1}+2 x_{2}+3 x_{3}+4 x_{4}$

S.T. $\quad-4 x_{1}+x_{2}-2 x_{3}-3 x_{4} \geq 1$

$x_{1}, x_{2}, x_{3}, x_{4} \geq 0$ 
Hence $A_{11}=[-4]$, given $x_{1}=-\frac{1}{4}$, is not feasible

$A_{21}=[1]$, given $x_{2}=1, \mathrm{z}=2$

$A_{31}=[-2]$, given $x_{3}=-\frac{1}{2}$ is not feasible

$A_{41}=[-3]$, given $x_{3}=-\frac{1}{3}$ is not feasible

And the optimal solution is $x_{1}=0, x_{2}=1, x_{3}=0, x_{4}=0$, and $z=2$.

\section{Conclusion}

The proposed method solve LPP without change the number of variables in the program and the optimal solution only dependent on the sub matrix which satisfy the constraints and gives the optimal value to the objective function. If all sub matrices are singular then, the solution is unbounded. If the choses sub matrix is singular then, the result from it is not feasible. The proposed method cannot solve the mixed LPP.

\section{References}

1. Hamdy A. Taha, 2016, operations research An Introduction, Tenth Edition, amazon

2. Kartono, 1994, TeoriPermainan, Andi Offset Yogyakarta

3. Sioshansi, Ramteen, Conejo, \& Antonio J., 2017, Optimization in Engineering Models and Algorithms, Springer

4. Catherine L., 2008, Linear Programming: Theory and Application, University of Texas 